This item was submitted to Loughborough's Research Repository by the author.

Items in Figshare are protected by copyright, with all rights reserved, unless otherwise indicated.

\title{
Driver sleepiness : comparisons between young and older men during a monotonous afternoon simulated drive
}

PLEASE CITE THE PUBLISHED VERSION

http://dx.doi.org/10.1016/j.biopsycho.2012.01.002

PUBLISHER

(C) Elsevier B.V.

VERSION

AM (Accepted Manuscript)

LICENCE

CC BY-NC-ND 4.0

\section{REPOSITORY RECORD}

Filtness, Ashleigh J., Louise A. Reyner, and James A. Horne. 2019. "Driver Sleepiness : Comparisons Between Young and Older Men During a Monotonous Afternoon Simulated Drive". figshare. https://hdl.handle.net/2134/15400. 
This item was submitted to Loughborough's Institutional Repository (https://dspace.lboro.ac.uk/) by the author and is made available under the following Creative Commons Licence conditions.

\section{creative
commons}

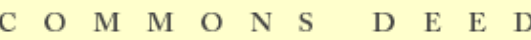

Attribution-NonCommercial-NoDerivs 2.5

You are free:

- to copy, distribute, display, and perform the work

Under the following conditions:

Attribution. You must attribute the work in the manner specified b the author or licensor.

Noncommercial. You may not use this work for commercial purposes.

No Derivative Works. You may not alter, transform, or build upon this work.

- For any reuse or distribution, you must make clear to others the license terms of this work.

- Any of these conditions can be waived if you get permission from the copyright holder.

Your fair use and other rights are in no way affected by the above.

This is a human-readable summary of the Leqal Code (the full license).

\section{Disclaimer 만}

For the full text of this licence, please go to: http://creativecommons.org/licenses/by-nc-nd/2.5/ 


\title{
Driver sleepiness - comparisons between young and older men during a monotonous afternoon simulated drive
}

Ashleigh J Filtness Ph. ${ }^{1,2_{*}}$, Louise A Reyner Ph. $\mathrm{D}^{1}$ \& James A Horne Ph. $D^{1}$

\author{
1. Sleep Research Centre \\ Loughborough University \\ Leicestershire LE11 3TU, UK \\ Phone+ 44(0)1509-22 8225 \\ $\mathrm{Fax}+44(0) 1509-228480$
}

2. Now at: Accident Research Centre

Monash University

Clayton 3188, Australia

Phone: +61 399020198

Fax: +61 399054363

ashleigh.filtness@monash.edu

*corresponding author

$26^{\text {th }}$ June 2011 


\begin{abstract}
Young male drivers feature predominately in road crash statistics, especially where the driver has fallen asleep. One possibility is that they are more vulnerable to the effects of sleep loss compared with older men. We assessed the effect of normal night sleep versus prior sleep restricted to $5 \mathrm{~h}$, in a counterbalanced design, on prolonged afternoon driving in 20 younger (av. 23y) and 20 older (av. 67y) healthy men who drove a full size, real car simulator under monotonous 'motorway' conditions for $2 \mathrm{~h}$ during the 'afternoon dip'. Driving was monitored for sleepiness related lane deviations, EEGs were recorded continuously and subjective ratings of sleepiness taken every $200 \mathrm{sec}$ throughout the drive. Following normal sleep there were no differences between groups for any measure. After sleep restriction, and compared with the older group, the younger drivers showed significantly more sleepiness-related deviations, greater $4-11 \mathrm{~Hz}$ EEG power, and a near significant increase of subjective sleepiness. Correlations between the EEG and subjective measures were highly significant for both groups, indicating good self-insight into increasing sleepiness. This study confirms the greater vulnerability of younger drivers to sleep loss under prolonged driving, even during the early afternoon.
\end{abstract}

Key words: sleepiness, driving ability, age effects, road safety, driving simulator 


\section{INTRODUCTION}

Studies of drivers falling asleep at the wheel usually involve young adults. This is largely because fatal road crash statistics worldwide show that they comprise the group most liable to be involved eg. USA - [1,2]; Israel - [3]; UK - [4]; Sweden - [5]. One reason is that they may be more vulnerable to sleep loss than older people, owing to a greater depth and intensity (more stage 4) of their sleep cf.[6-8]; thus sleep loss may be more profound.

There have been several reports of young adults being more vulnerable to sleep loss, such studies have utilised simple performance indices, such as the PVT and often use a total sleep deprivation protocol $[6,9,10]$. Few have involved realistic driving situations. The problems of using a simple vigilance task to assess the impact of sleep loss is demonstrated by Blatter et al [11] who used a $40 \mathrm{~h}$ sleep deprivation protocol in older adults. A significant decline in performance at a maze planning task was reported. This involved similar cognitive planning requirements of driving. The PVT was also completed but no impairment was found, suggesting the PVT to be too simple a task to detect the subtle cognitive impairment which is relevant to driving.

Heightened vulnerability to sleep loss in the young has also been demonstrated using night time driving task protocols. Campagne et al [12] monitored three age groups (20-30y, 40-50y and 60$70 \mathrm{y}$ ) driving for an average of $2 \mathrm{~h} 49 \mathrm{~min}$, on a full-size simulator, under monotonous conditions at night. Run off the road driving errors were found to be more common in younger participants than either of the two older groups. Another driving simulator study also investigating night time driving by Lowden et al [13] involving young (18-24y) and older (55-64y) participants driving a full-size car simulator for $45 \mathrm{~min}$, between $02: 30$ and 04:00h. Findings clearly showed the younger group to be sleepier, as determined by EEG and subjective responses, (using a modified Karolinska Sleepiness Scale - KSS - Reyner \& Horne, [14]). Although lane drifting was similar for both groups, the authors noted that this drive duration was fairly short. Sagaspe et al [15], conducted a night time on road driving study, with younger and older participants, investigating two countermeasures to sleepiness: a nap and caffeine. Although the aim of this study was to assess the effectiveness of the countermeasures for each group, under placebo conditions $75 \%$ of older participants were able to maintain performance to that in the daytime compared with only $25 \%$ of younger participants. In this study the older participants were middle aged, $40-50 y$, not as old as the older groups used in other studies.

In the context of age effects and driving performance two areas under investigation are the affect of sleep restriction and day time driving. It is important that a protocol using sleep restriction (as opposed to sleep deprivation) is investigated because sleep deprivation and sleep restriction have been shown to have differing effects on task performance [16]. Road crash statistics show two peaks in sleep related incidents one in the early hours of the morning and the other in the afternoon [17]. Such an age comparison driving task protocol has also not been conducted in the 
early afternoon. The night time peak is caused mainly by younger drivers and they daytime peak mainly by older drivers. The driving studies mentioned above all examine driver sleepiness during night time driving and find younger participants to be more vulnerable to sleep restriction. However, similar comparisons have not been made at the time of day where older drivers are more likely to have sleep related incidents.

A very recent driving study of ours, focussing on older men successfully treated for obstructive sleep apnoea [18], included a control group of 20 age-matched healthy men (see below). We are able to compare this latter group with young male drivers, from our other studies, involving identical protocols and on the same real car simulator (see below). All participants drove for $2 \mathrm{~h}$ during the early afternoon 'dip', under conditions of a normal prior night's sleep vs sleep restricted to $5 \mathrm{~h}$ (designed to worsen the dip). Measurements comprise lane drifting, EEG and subjective sleepiness. Data for the young drivers comes from 12 participants from Horne et al. [19] plus 8 others from an unpublished study.

A topic of current interest is the extent to which sleepy drivers are aware of their sleepiness when driving cf. [20]. We [21,22] have previously shown very close ('on-line') associations between subjective and EEG measures (4-11 Hz power - see below) in young drivers, to the extent that they are quite aware of increasing sleepiness. We also report, here, the extent of this association for older drivers, which is a hitherto unreported finding. Finally, we recruited male drivers as in the UK, men are responsible for the majority (90\%) of sleep related collisions [eg. 4,23], although it is acknowledged that men have a higher exposure to driving.

\section{METHOD}

\subsection{Participants}

Two groups of healthy male participants were recruited, 20 older participants, with a mean age $66.6 y(52-74 y)$ and 20 younger participants with a mean age of 22.7 (20 - 26y). All were good sleepers with the younger participants having slightly longer average usual sleep duration by actigraphy (503min compared with $468 \mathrm{~min}$ ), all scored $<10$ on the Epworth Sleepiness Scale (ESS) [24].

The older participants were recruited via advert and initially screened by postal questionnaire and phone. Initial screening removed those with estimated BMIs $>28$, who drove for $<3$ her week or lived further than $40 \mathrm{~km}$ of our research centre. Remaining potential participants were invited to our Centre and further questioned on illnesses (especially heavy snoring and other signs of obstructive sleep apnoea [OSA]), medications liable to affect sleep, and habitual sleep characteristics, as well as for coffee consumption ( $<4$ cups daily). Height and weight were measured to ensure a BMl<28 (as BMI greater than this represents increased risk of OSA).. 
The younger participants were recruited by advert at the university and screened by interview and by the same methods as for the older participants. They were healthy, medication-free, with a mean BMI of 23.0 (range 20-27). All were experienced drivers (having driven for over 2y, for more than $3 \mathrm{~h}$ per week).

A 30 min familiarisation drive in the simulator (below) was completed by all participants prior to the experimental days.

All participants had the procedures fully explained, signed consent forms, and received either a gift voucher or were paid the equivalent value on completion of the study. The study had full approval of the University's Ethical Committee. For both experimental phases (see below), all participants were collected from and returned to their home via taxi.

\subsection{Design and Procedure}

Participants underwent a $2 \mathrm{~h}$ simulator drive following two experimental conditions, following i) normal sleep ii) sleep restriction to $5 \mathrm{~h}$ by delayed bed-time. Test days were completed in a counterbalanced design, with each condition 1-2 weeks apart.

To ensure compliance with sleep instructions, participants wore wrist actimeters (Cambridge Neurotechnology, UK) for three nights prior to each experimental day, when they also kept daily logs of: estimated sleep onset, and morning wakening and rising times. No alcohol was consumed $36 \mathrm{~h}$ prior to each test session, and nil caffeine after 18:00h the evening before. Participants refrained from eating after 10:00h on the morning of the drive. On arrival at the laboratory, at 13:00h, participants were given a light lunch. Actimeters were downloaded to verify that they had complied with the previous night's sleep requirements. At 13:15h electrodes were applied and they went to the simulator (see below) at 13:50h, to be given $10 \mathrm{~min}$ to settle into the car. The $2 \mathrm{~h}$ continuous drive began at 14:00h.

Participants were instructed to drive in the left hand lane (unless overtaking), (as per U.K. road rules) at a speed appropriate for the road and at which they were able to maintain control of the vehicle. During the drive the investigator was in the room at all times in case of any problem, but there was no communication between investigator and participant once the drive had begun.

\subsection{Apparatus}

\subsubsection{Car Simulator}

This comprises an immobile car with a full-size, interactive, computer generated road projection of a dull monotonous dual carriageway; each having two lanes. The image was projected onto a 2.0m $x 1.5 \mathrm{~m}$ screen, located $2.3 \mathrm{~m}$ from the car windscreen. The road had a hard shoulder and simulated auditory 'rumble strips' (incorporated into white lane markings) either side of the 
carriageway, with long straight sections followed by gradual bends. 'Crash barriers' are located either side, beyond the rumble strips. Slow moving vehicles are met occasionally, that require overtaking (to avoid collision).

Driving incidents: Lane drifting is the most common manifestation of sleepy driving, when all four wheels are out of the driving lane (lane departure) this was identified as a driving 'incident'. Splitscreen video footage of the roadway and driver's face (filmed by an unobtrusive infrared camera) was scrutinised and enabled the cause of the incident to be determined. Those caused by sleepiness (e.g. eye closure, eyes rolling upwards or vacant staring ahead) were logged as 'sleeprelated'. As a further check for the latter, the EEG and electrooculogram (EOG - see below) were examined respectively for alpha/theta intrusions and confirmation of any 'eye rolling'. Non-sleep related incidents (driver distraction, fidgeting or looking around) were excluded, therefore all results refer to sleep related incidents only.

\subsubsection{Subjective Sleepiness}

Every 200s during the drive, participants were verbally prompted by the computer system ('sleepcheck') to report their subjective sleepiness on the 9-point Karolinska Sleepiness Scale (KSS): 1=extremely alert, 2=very alert, 3=alert, 4=rather alert, 5=neither alert nor sleepy, 6=some signs of sleepiness, 7=sleepy, no effort to stay awake, 8=sleepy, some effort to stay awake, 9=very sleepy, great effort to keep awake, fighting sleep. The scale was located on the car's dashboard and continuously visible to the driver. This prompting and its response quickly became routine for the driver.

\subsubsection{EEG and EOG}

Electrodes were attached for two channels of EEG, with inter-electrode distances maintained by using the '10-20 EEG montage' (main channel $C_{3}-A_{1}$, backup channel $C_{4}-A_{2}$ ). There were two EOG channels (electrodes $1 \mathrm{~cm}$ lateral to and below left outer canthus and $1 \mathrm{~cm}$ lateral to and above right outer canthus; both referred to the centre of the forehead). EEGs and EOGs were recorded using "Embla" (Flaga Medica Devices, Iceland) and spectrally analysed using "Somnologica" (Flaga) in 4s epochs. EEG low and high band-pass filtering at $>20 \mathrm{~Hz}$ and $<4 \mathrm{~Hz}$ removed slow eye movements and muscle artefact, any remaining interferences by motoric movement (body, jaws etc) were visually removed.

In these circumstances, greater EEG power in the alpha $(8-11 \mathrm{~Hz})$ and theta $(4-7 \mathrm{~Hz})$ ranges reflect increased sleepiness. Power in the combined range $(4-11 \mathrm{~Hz})$ was averaged in one minute epochs. To accommodate for individual differences, and to allow comparisons between conditions, each individual's power in these ranges was standardised, by taking the difference between each minute's epoch and the individual's mean value over the first 30min of baseline data, which was then divided by the standard deviation around the mean of that 30min of data cf. [19] 


\subsection{Statistical Analysis}

Mixed model, repeated measures analysis of variance (ANOVA) were utilised, with one betweensubject (groups) and two within subject factors: i) Condition - two levels: normal sleep and sleep restriction; ii) Duration of driving - four levels: 0-30 $\mathrm{min}, 30-60 \mathrm{~min}, 60-90 \mathrm{~min}$ and 90-120min. Huynh-Feldt $(\varepsilon)$ adjustments were used if the assumption of sphericity was not met. Where appropriate square root transformations corrected for skewed driving incident raw data. For all measures, data was collapsed into 30 minute epochs for statistical analysis

\section{RESULTS}

\subsection{Driving Incidents - Figure 1.}

There is a significant effect for sleep condition on the number of driving incidents, showing both groups to have more incidents following sleep restriction $[F(1,38)=27.67, p=0.000, \varepsilon=1]$. A significant condition by group interaction showed the younger participants were more impaired by sleep restriction than the older participants $[F(1,38)=9.92, p=0.003, \varepsilon=1]$. There was also a significant time effect, with number of incidents increasing for both groups with time on task [F (2.6, $99.0)=4.8, p=0.005, \varepsilon=0.87$ ]. Although from the graph there is an apparent greater increase in number of driving incidents by younger participants between the first and second 30 minutes of the drive there was no significant group and time interaction.

\subsection{KSS - Figure 2.}

The affect of sleep condition was significant on subjective sleepiness, as would be expected [F $(1,38)=46.55, p=0.000, \varepsilon=1]$. There was a near-significant trend for a sleep condition by group interaction $[F(1,38)=3.41, p=0.073, \varepsilon=1]$, suggesting the younger participants to report feeling more sleepy following sleep restriction than the older participants. There was also a significant effect of time $[F(2.06,78.09)=39.87, p=0.000, \varepsilon=0.69]$ again showing time on task to have an increased affect on sleepiness for both groups, but no significant group by time interaction.

\subsection{EEG - Figure 3.}

There was no overall significant effect of sleep condition on standardised EEG activity (alpha and theta), but there was a significant sleep condition by group interaction $[F(1,36)=5.03, p=0.031, \varepsilon$ $=1$ ], whereby the younger participants showed more EEG determined sleepiness following sleep restriction than the older participants. The effect of time was also significant $[F(1.84,66.29)=$ 18.71, $p=0.000, \varepsilon=0.61$ ] showing sleepiness to increase throughout the drive's for both groups, but with no other significant interactions.

\subsection{Correlations between KSS and EEG_- Figures $4 a$ and $b$.}


For both groups under sleep restriction conditions only the mean EEG data were collapsed into 200 sec epochs, smoothed by a three point running average and then compared with KSS group means per $200 \mathrm{sec}$. For both groups the correlations were highly significant, particularly for the older group [Older $-r=0.90 ; p<0.000 \mathrm{df}=35 ;$ Young $-r=0.72 ; p<0.001 \mathrm{df}=35$ ]. Also shown, superimposed on the graphs are $5^{\text {th }}$ order polynomial fits.

\section{DISCUSSION}

Following sleep restriction, and compared with the younger participants, older participants had significantly less sleep related driving incidents, lower EEG power in the 'sleepy' range, and a near- significant lower level of subjective sleepiness during an afternoon drive. These findings point to older drivers being less vulnerable to sleep loss related driving impairments, which are generally in agreement with previous night time driving studies $[12,13,15]$.

For the time of day the study was conducted it might have been expected that the older participants would have more incidents, and therefore reflective of real road traffic accident data reports [17]. However, the reverse was found, this is an interesting finding as the older participants in the current work were having sleep related incidents, all be it to a lesser extent than the younger participants. In light of the current findings (that younger participants are more vulnerable to sleep restriction than older participants in the early afternoon) the traffic data may in fact reflect a disproportion over representation of older drivers being on the roads at this time of day and similarly a greater number of young drivers being on the road in the early hours of the morning making them overrepresented in these statistics.

Out of lane incidents were the key measure of driving performance used in the current work to demonstrate that the driving performance of younger participants is significantly more affected by sleep restriction than that of older participants. We also demonstrate a significantly higher level of EEG 'sleepiness' in the younger participants and suggest the impaired driving performance is shown to be as a result of greater sleepiness. Campagne et al [12] reported younger participants to drive faster than older participants and that excessive speed errors were positively correlated with exiting the road lane. Driving speed was not considered in the current work but it is possible that as well as the lower vulnerability to sleep restriction (demonstrated by EEG activity) older participants may also have been able to limit the number of driving incidents they had by driving at a more cautious speed.

Driving is a complex task requiring various cognitive skills, Frey et al [25] reported sleep deprivation to impair cognitive task performance at various measures of brain function differently for different participants. In a battery of cognitive tasks, they found all participants to have an overall declined in cognitive ability following sleep deprivation but that the particular tests showing most impairment varied between people. It is not possible to tell from the current study if the 
younger participants all had worse performance due to the same type of impairment or whether a variety of brain functions were impaired. In the practical sense of road safety it is not important which specific cognitive functions are impaired in which people, only whether or not the end output of controlling a vehicle can be maintained, and in this measure younger participants have greater difficulty than older participants.

Important to road safety is a person's ability to recognise when they are sleepy in order for the individual to be able to act on this knowledge and stop driving before an incident occurs. We report here that both our groups had good insight into their increasing sleepiness following sleep restriction, showing close associations between subjective and EEG measures of sleepiness. We have reported this previously in younger participants and now are able to extend this finding to older participants.

This being a simulator-based study, it could be argued that the findings are of limited comparability with real driving conditions. However, whilst it is ethically difficult to conduct such studies on real roads, it is likely that without the awareness of danger, apparent under real conditions, simulator studies are liable to reflect greater and more rapid effects of sleepiness. Nevertheless, ours was a full size, and realistic car simulator, and comparisons between real driving and laboratory studies, even with the latter utilising simpler simulators than ours, have shown that the two methods are comparable [26], albeit somewhat exaggerated in the laboratory. The length of the drive was chosen to reflect the recommendation of safe length of driving that is recommended by the UK government, the fact that both groups were having sleep related incidents throughout the drive may demonstrate the exaggerated laboratory affect of driving simulators.

Our findings are limited to male drivers but as stated in the introduction, males are over represented in on road sleep related incidents $[4,23]$ so are a relevant group to study. The younger participants had slightly longer usual sleep, where both groups were restricted to 5 hours sleep in the experimental conditions it is possible that some of the increased impairment is due to the younger participants being on average 35min more sleep deprived than the older participants. This limitation is acknowledged and future research may wish to take account of this and restrict sleep to a similar degree between participants. However, the current study design enables conclusions to be drawn in comparison of differing age groups experiencing the same amount of sleep.

The greater vulnerability of the younger participants to sleep restriction potentially puts them at a greater driving risk under these circumstances, and may help explain the relatively high proportion of young men being responsible for serious sleep related road crashes [17,27]. Although the peak in sleep related crashes in the afternoon by older drivers cannot be explained from the current study it is speculated that this may be a reflection of the distribution of driver age on the road at this time and is not due to heightened vulnerability to sleep restriction at this time. 


\section{ACKNOWLEDGEMENTS}

We would like to acknowledge and thank Dr Pauline Barrett for the collection and initial analysis of data from the younger participants. 


\section{REFERENCES}

[1] Langlois PH, Smolensky MH, Hsi BP, Weir FW. Temporal patterns of reported single-vehicle car and truck accidents in Texas USA during 1980-1983. Chronobiol Int. 1985; 2:131-146.

[2] Pack Al, Pack AM, Rodgman E, Cucchiara A, Dinges DF, Schwa, CW. Characteristics of crashes attributed to the driver having fallen asleep. Accid Anal Prevent 1995; 27: 769-775.

[3] Zomer J, Lavie P. Sleep-related automobile accidents - when and who ? In: Horne JA editor Sleep '90, Bochum - Pontenagel Press, 1990, p.448-451.

[4] Horne JA, Reyner LA. Sleep related vehicle accidents, Br Med J 1995; 310: 565-567.

[5] Akerstedt T, Kecklund G. Age, gender and early morning highway accidents. J Sleep Res 2001;10:105-10

[6] Adam M, Retey JV, Khatami R. Age-related changes in the time course of vigilant attention during 40 hours without sleep in men, Sleep 2006;,29: 55-57.

[7] Klerman EB, Dijk DJ. Age-related reduction in the maximal capacity for sleep--implications for insomnia. Curr Biol. 2008;18:1118-23

[8] Dijk DJ, Groeger JA, Stanley N, Deacon S. Age-related reduction in daytime sleep propensity and nocturnal slow wave sleep Sleep. 2010;,33:211-23.

[9] Philip P, Taillard J, Sagaspe P, Valtat C, Sanchez-Ortuno M, Moore N, Charles A, Bioulac B. Age, performance and sleep deprivation. J Sleep Res 2004;13:105-10.

[10] Duffy JF, Willson H, Wang W, Czesiler C. Healthy older adults better tolerate sleep deprivation than young adults, JAGS 2009; 57:1245-1251

[11] Blatter K, Opwis K, Munch M, Wirz-Justice A, Cajochen C. Sleep loss related decrements in planning performance in healthy elderly depend on task difficulty. J. Sleep Res 2005; 14:409-417.

[12] Campagne A, Pebayle T, Muzet A. Correlation between driving errors and vigilance level: influence of the driver's age. Physiology \& Behavior 2004; 80:515-524.

[13] Lowden A, Anund A, Kecklund G, Peters B, Akerstedt T. Wakefulness in young and elderly subjects driving at night in a car simulator. Accid Anal Prev.,2009;,41:1001-7.

[14] Reyner LA, Horne JA Suppression of sleepiness in drivers: combination of caffeine with a short nap. Psychophysiol,1997; 34: 721-725.

[15] Sagaspe P, Tailard J, Chaumet G, Moore N, Bioulac B, Philip P. Aging and nocturnal driving: better with coffee or a nap? A randomised study. Sleep. 2007; 30:12: 1808 - 1813. 
[16] Belenky G, Wesensten NJ, Thorne DR, Thomas ML, Sing HC, Redmond DP, Russo MB, Balkin TJ. Patterns of performance degradation and restoration during sleep restriction and subsequent recovery: a sleep dose-response studyJ. Sleep Res. 2003: 12;1-2.

[17] Flatley D, Reyner LA, Horne JA Sleep related crashes on sections of different road types in the UK (1995-2001) 2004. UK Department for Transport - Road Safety Research Report No 52

[18] Filtness A, Reyner LA, Horne JA. Moderate sleep restriction causes greater impairment to monotonous driving in treated OSA participants compared with controls. unpublished

[19] Horne JA, Reyner LA, Barrett PR. Driving impairment due to sleepiness is exacerbated by low alcohol intake, Occ Environ Med 2003; 60: 689-692

[20] Anund A, Akerstedt T. Perception of sleepiness before falling asleep. Sleep Med 2010;11: 743-744

[21] Horne, JA, Reyner LA Counteracting driver sleepiness: effects of napping, caffeine and placebo. Psychophysiol 1996; 33: 306-309

[22] Horne JA, Baulk SD Awareness of sleepiness when driving. Psychophysiol 2003; 41: 161-165

[23] Maycock G. Sleepiness and driving: the experience of U.K. car drivers. Accid Anal Prev.1997; 29:453-62.

[24] Johns MW A new method for measuring daytime sleepiness: the Epworth Sleepiness Scale. Sleep, 1991;14: 540-546.

[25] Frey DJ, Badia P, Wright KP. Inter and intra individual variability in performance near the circadian nadir during sleep deprivation. J Sleep Res. 2004; 13: 305-315.

[26] Philip P, Sagaspe P, Taillard J, Valtat C, Moore N, Akerstedt T, Charles A, Bioulac B. Fatigue, sleepiness, and performance in simulated versus real driving conditions Sleep 2005; 28:1511-6.

[27] Groeger J. Youthfulness, inexperience and sleep loss the problems young drivers face and those they pose for us. Injury Prevent 2006;12 (Suppl I):19-i24 


\section{FIGURE LEGENDS}

\section{Figure 1}

Group mean driving incidents (s.e. bars) per 30 min of drive for both groups and sleep conditions. Sleep loss significantly worsened driving in the younger group.

\section{Figure 2}

Subjective sleepiness (KSS) - group means per 200sec for groups and conditions. The greater effect of the sleep loss on the younger group was almost significant.

\section{Figure 3}

EEG alpha+theta power (indicative of increasing sleepiness), per minute for both groups and conditions. Data have been smoothed by a 3-point running average. Sleep loss led to a significant increase in the younger group.

\section{Figure $4 a$ and $b$}

Smoothed EEG alpha+theta power with polynomial fit, plotted with KSS for older (top graph) and younger drivers (bottom). There was a highly significant correlation between both variables. 
Figure 1

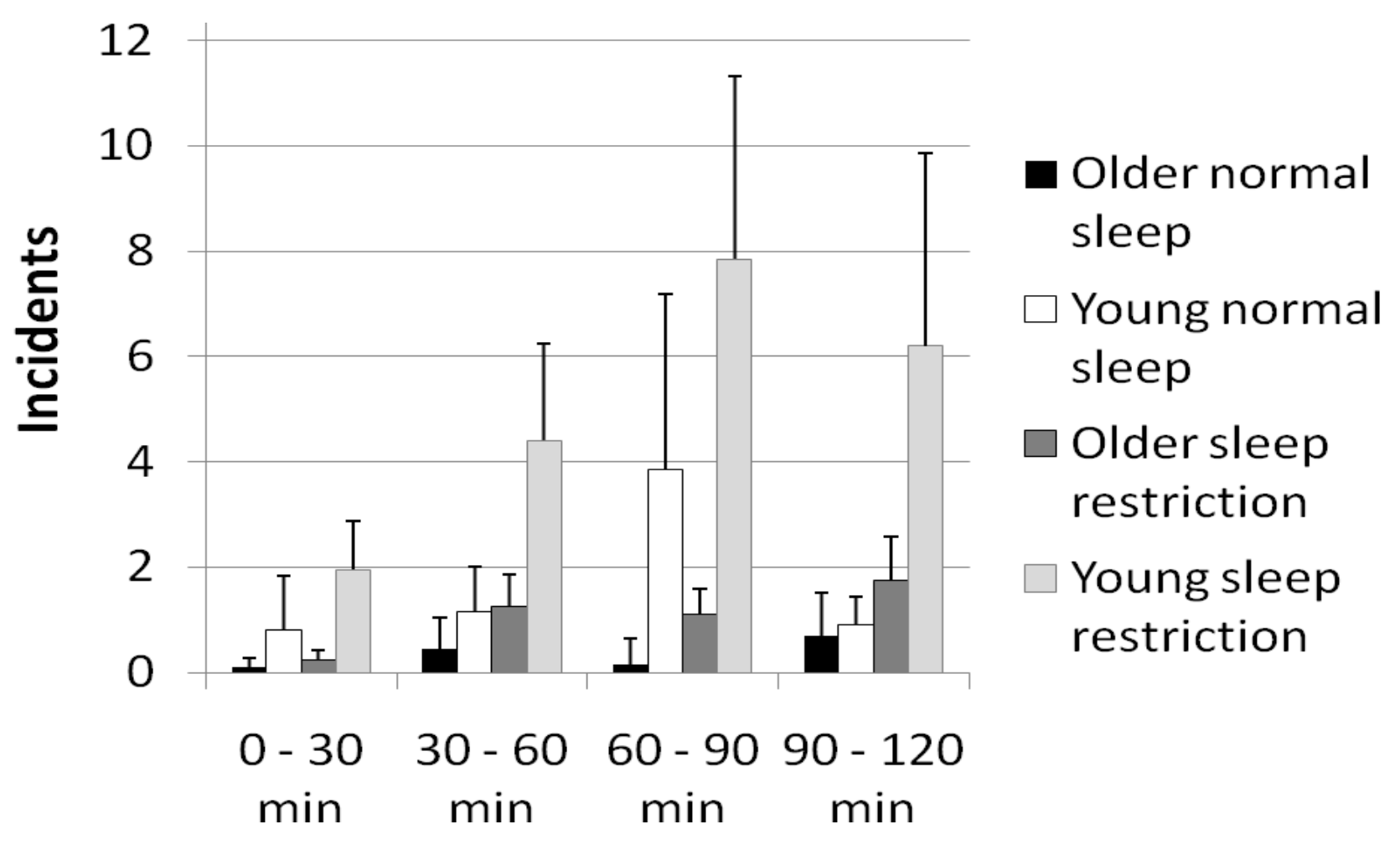


Figure 2

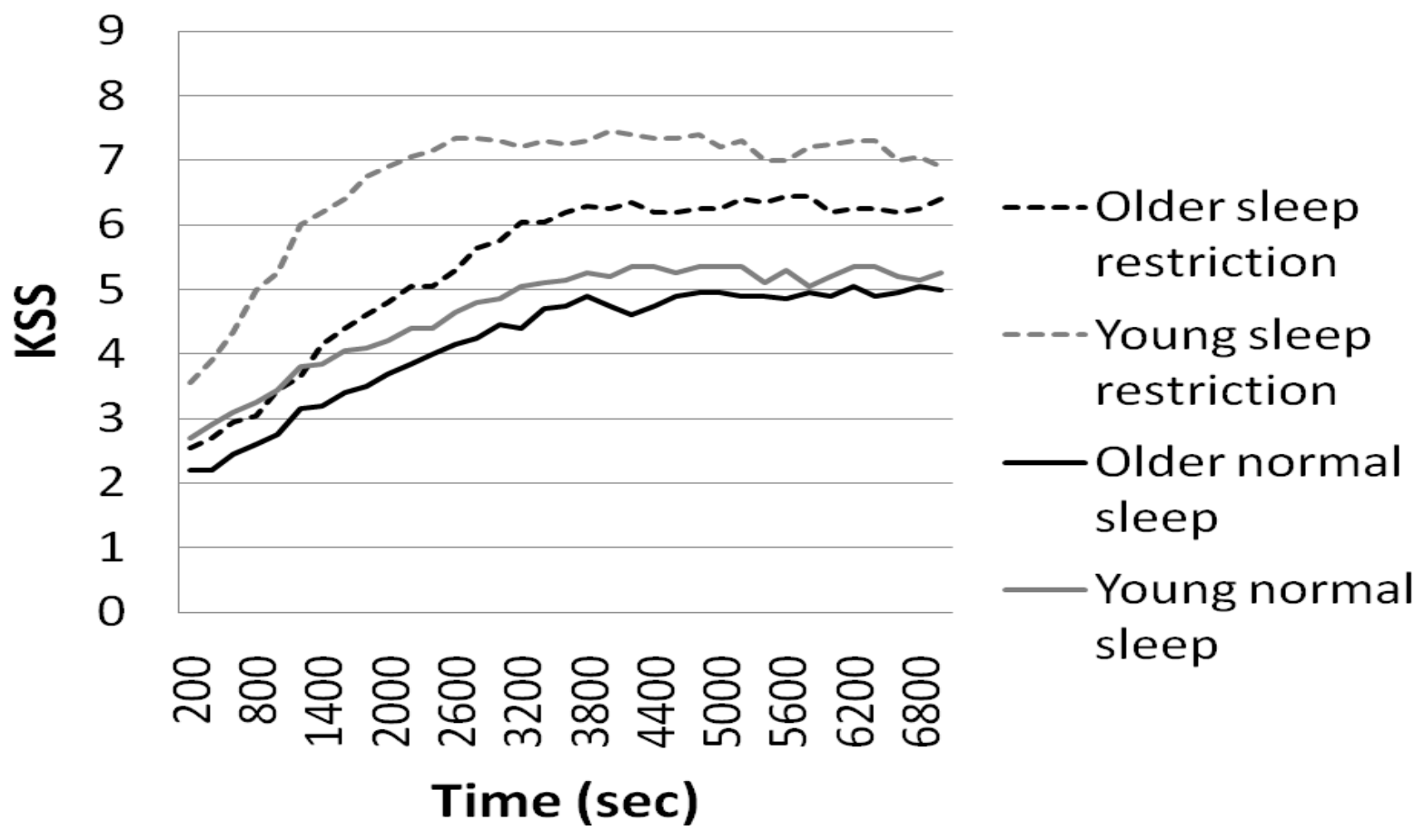

Figure 3

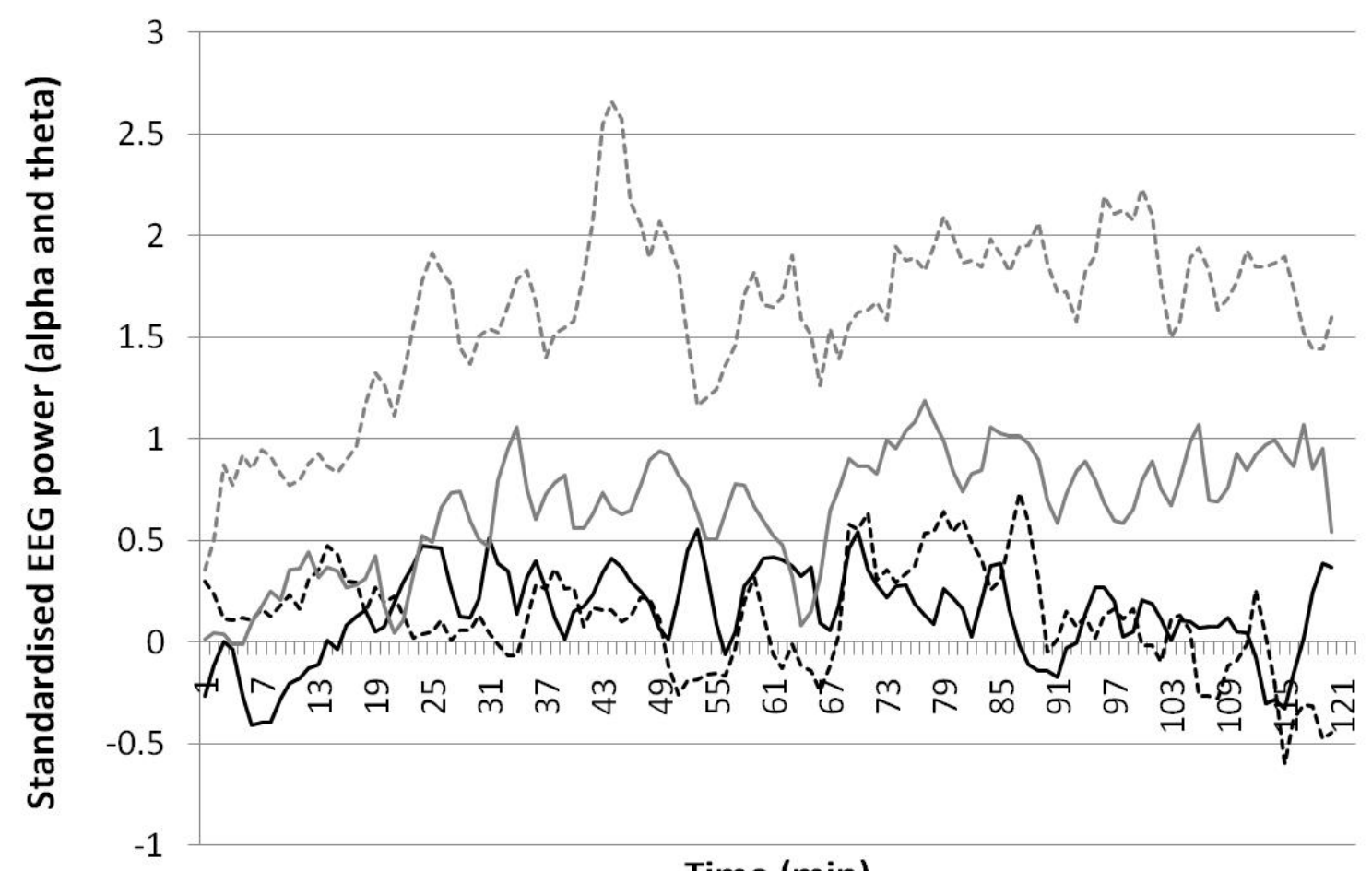

Time (min)

-Old normal sleep 3PRA $\quad$----Old sleep restriction 3PRA

-Young normal sleep 3PRA ----Young sleep restriction 3PRA 
Figure $4 a$

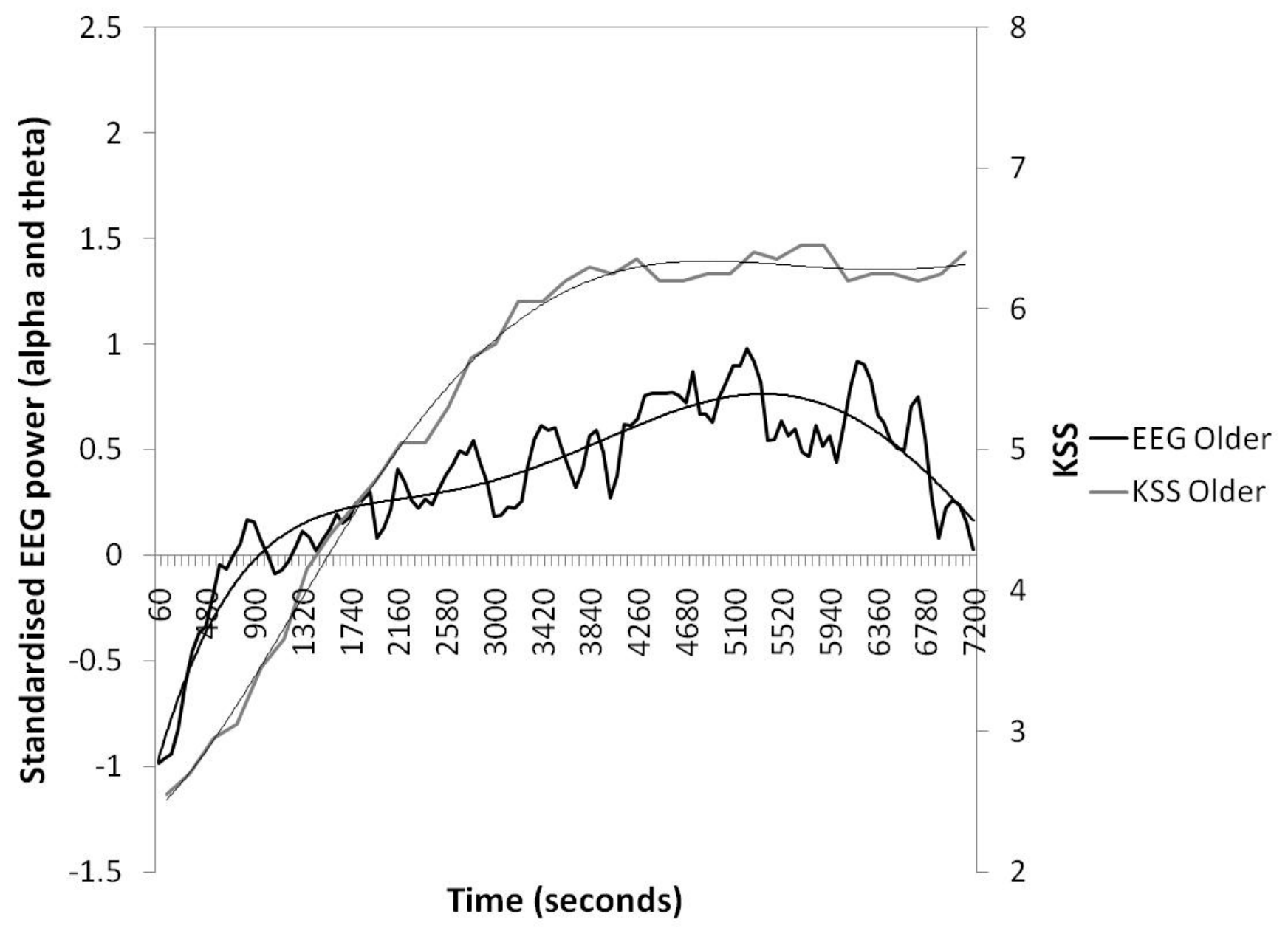


Figure 4b

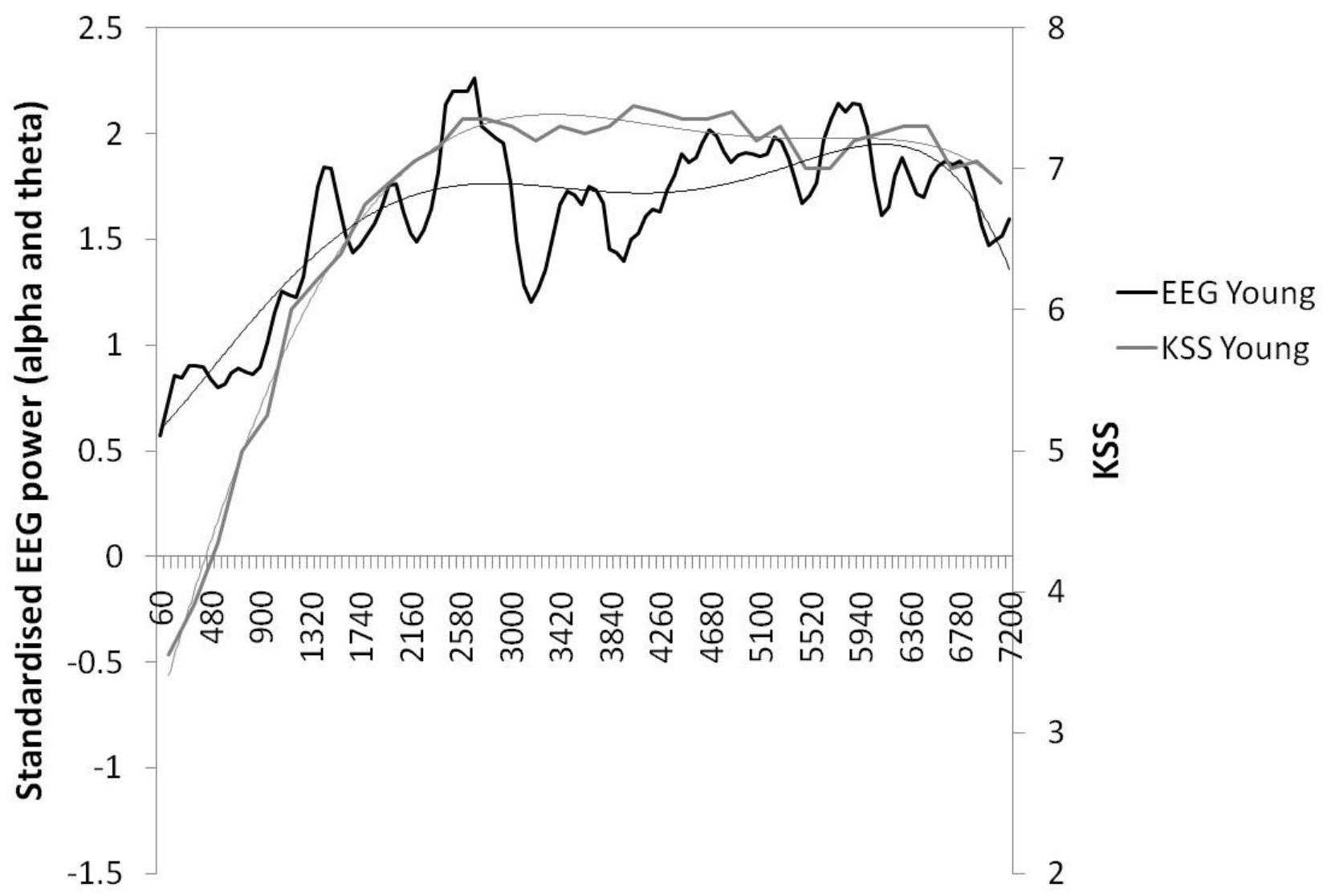

Time (seconds) 